\title{
A importância do estudo do tracking (estabilidade e previsão) em delineamentos longitudinais: um estudo aplicado à epidemiologia da actividade física e à performance desportivo-motora
}

\author{
José A. R. Maia \\ Rui G. da Silva \\ André Seabra \\ Faculdade de Ciências do Desporto e de Educação Física \\ Universidade do Porto, Portugal
}

\section{RESUMO}

Esta pesquisa pretende demonstrar a importância do estudo do tracking em investigação epidemiológica e da performance desportivo-motora. A partir de dois conjuntos de dados é apresentado o problema, a sua relevância e essência analítica. No primeiro estuda-se o tracking da participação em actividade física (i.e., práticas desportivas generalizadas) a partir de informação proveniente de 588 sujeitos seguidos longitudinalmente dos 12.7 aos 17.7 anos. Foi utilizado um modelo quasi-simplex, que evidenciou a elevada estabilidade da prática desportiva, bem como o valor moderado a elevado das trajectórias interindividuais. No segundo estudo recorremos a informação proveniente de 12 saltadores em comprimento, a partir da sua melhor marca em seis anos consecutivos. Recorreu-se ao conceito de canalização a partir dos procedimentos propostos por Cohen, e Foulkes e Davies. Os resultados salientaram um fraca estabilidade da performance no tempo, bem como o seu carácter não-linear. Em conclusão há que salientar três aspectos fundamentais: o primeiro radica na necessidade do uso de novos procedimentos para pesquisar o fenómeno do tracking; o segundo sugere a forte estabilidade e tracking moderado a elevado na prática desportiva ao longo da adolescência; o terceiro salienta a fraca canalização da performance dos saltadores, revelando a ausência de estabilidade.

Palavras-chave: Tracking, modelos, simplex, actividade física, performance.

\author{
Vítor P. Lopes \\ Instituto Politécnico de Bragança, Portugal
}

\section{ABSTRACT}

The importance of tracking (stability and prediction) in longitunal designs: an applied study to the epidemiology of physical activity and sports performance

This study aims at the presentation of the tracking phenomena in epidemiological and motor performance research.

The problem of tracking, its essence and importance were presented based on two sets of data. The first one was related to physical activity (i.e., sports practise) of 588 youngsters followed longitudinally for 6 years, starting at the age of 12.7 years and ending at 17.7 years. A quasi-simplex model was used, and showed the high stability of sports practise, as well as the moderate to high tracking.

In the second study, information came from the best performance of 12 long jumpers in six consecutive years. This data was analysed according to the concept of canalisation, using the procedures suggested by Cohen, and Foulkes and Davies. Results showed a low stability of performance over time, as well as its non-linear behaviour.

In conclusion, three aspects deserved main attention: the first one related to the use of novel approaches to the study of tracking; the second one showed the high stability of sports practise, as well as the moderate to high tracking; the third one evidenced the low canalisation of the performance of the long jumpers due to its low stability.

Key Words: Tracking, modeling, simplex, physical activity, performance 


\section{INTRODUÇÃO}

A interpretação, significado e alcance do estudo da estabilidade e da mudança de características ou traços mensuráveis, contínuos ou discretos, de atletas de níveis distintos de rendimento são do maior interesse dos pesquisadores das Ciências do Desporto. Estabilidade, mudança e previsão exigem, necessariamente, informação de natureza longitudinal, supondo, ou não, que os pontos de observação e registo dos traços em estudo sejam equidistantes no tempo 5, 44.

O termo tracking contém, em si mesmo, uma dupla noção: a primeira refere-se à distância constante entre um par de rodas, e a segunda contempla a possibilidade em seguir uma pista que deixa marcas visíveis no terreno 11,39. Deste carácter bi-fronte do tracking emergem quatro pontos chave: (1) presença de indicadores no terreno, (2) estabilidade das pistas, (3) facilidade em seguir uma pista, e (4) antecipar pontos da pista a partir de outros previamente conhecidos. O tracking implica, pois, duas ideias que se interpenetram - estabilidade e previsibilidade 50 . Tracking é um termo genérico que pretende descrever um padrão regular de crescimento ou mudança numa colecção de padrões de crescimento. Kowalski e Schneiderman (1992) referem, por um lado, a ausência de uma definição universal para o termo, e por outro a ideia genérica do seu conteúdo - tendência de um indivíduo ou colecção de indivíduos em permanecer(em) num dado curso ou canal de crescimento ou mudança, reflectindo estabilidade no seu padrão de crescimento ou mudança.

O estudo do tracking é da máxima relevância se pretendermos atribuir significado próprio aquilo que é ou não estável nos sujeitos em função da história natural do seu desenvolvimento, de um dado programa de intervenção, ou de um tratamento específico. A título de mero exemplo ilustrativo, salientaremos aspectos centrais de alguns domínios de pesquisa onde a matéria do tracking é saliente:

- Biologia Humana, Antropologia Física e Auxologia Verificar o efeito do estatuto sócio-económico ou maturacional na variação do crescimento somático de uma população, ou verificar o grau de estabilidade do processo individualizado de crescimento somático.
- Biologia e Clínica

Determinar os efeitos de uma terapêutica hormonal no crescimento somático (contrastar efeitos distintos de terapias diferentes).

- Epidemiologia

Avaliar o padrão de crescimento somático de um sujeito relativamente aos valores de referência (identificar problemas de crescimento somático), ou inventariar o comportamento de grupos extremos relativamente ao factor de risco $\mathrm{x}$ ou variável $\mathrm{z}$. - Aprendizagem Motora

Verificar a magnitude e padrão de resposta de grupos de sujeitos submetidos a protocolos distintos de aprendizagem, ou avaliar o follow-up de sujeitos em condições distintas de aprendizagem.

- Treino Desportivo

Analisar o comportamento da performance individual ou grupos distintos de sujeitos submetidos a protocolos diferenciados de treino, ou inventariar a resposta a intervenções específicas de treino (estudos de caso).

Ainda que as metodologias disponíveis para abordar quantitativamente o fenómeno do tracking sejam diversificadas 24,39, foram Foulkes e Davies, em 1981, os primeiros autores a sugerirem a existência de duas correntes fundamentais de pensamento metodológico relativo à operacionalização desta noção:

- A primeira centra a sua atenção (1) no estudo das correlações entre medições sucessivas da(s) mesma(s) variável(eis) (i.e. auto-correlações), bem como de (2) regressão linear ou não-linear, sendo que daqui emerge a possibilidade de predição de valores futuros com base em observações prévias. Exemplos esclarecedores desta posição são evidentes em pesquisas epidemiológicas acerca do comportamento da tensão arterial 40 , de factores de risco da doença coronária 1 , actividade física 18,36, aptidão aeróbia 17 , ou em estudos na área da educação 6. Há um outro conjunto de autores (ver por exemplo 51) que estende a ideia da estrutura das auto-correlações (se estas evidenciarem estabilidade em que o valor de $r \geq 0.50$ ) à possibilidade de previsão. Aqui o enfoque é em estudos multivariados com recurso a generalized estimation equations e 
curvas de crescimento polinomial em dois estádios. Exemplos relevantes são os de Baumgartner e Roche (1988) acerca do padrão de adiposidade subcutânea, ou o de Twisk et al. (1996) relativo ao comportamento do colesterol e das lipoproteínas de alta densidade.

- A segunda corrente dirige o seu olhar para os seguintes aspectos: (1) o carácter inequívoco da distribuição dos valores monitorizados mudarem naturalmente com o tempo; (2) e os indivíduos "manterem as suas posições relativas", uns relativamente aos outros, em cada distribuição de valores e em cada ponto do tempo. Daqui que o problema nuclear a que se pretende dar resposta é precisamente o da definição precisa de posição relativa. A resposta tem sido diversificada e os procedimentos analíticos também são distintos, embora os resultados finais sejam idênticos $14,31,51$. As formas mais óbvias de solucionar a noção de posição relativa centram-se na ordem ou posição dos sujeitos (i.e., no cálculo de medidas de posição), no estudo do comportamento das posições dos sujeitos numa distribuição quantílica, ou no cálculo dos desvios da média em função do tempo 24 . Alguns exemplos desta abordagem estão consignados na pesquisa de Kelder et al. (1994) sobre o comportamento de adolescentes relativo aos seus hábitos tabágicos, de actividade física e de escolhas alimentares, ou ainda de Van Lenthe et al. (1994) no que concerne ao comportamento da tensão arterial em crianças e jovens. Um estudo fascinante é o de Park et al. (1997), acerca do fenómeno da ausência de canalização do peso e estatura durante a infância e adolescência.

Os dois estudos que iremos apresentar acerca do tracking provêm de domínios distintos de interesses: epidemiologia da actividade física e performance desportivo-motora. Nestes olhares diversificados sobre a dinâmica de um fenómeno interactivo - estabilidade, mudança e previsibilidade - recorreremos a modelos díspares de análise, por forma a salientar aos leitores algumas facetas do diamante polifacetado de olhares sobre a matéria. No primeiro recorreremos ao simplex de Markov. No segundo, ao Kappa de Cohen e ao gama de Foulkes e Davies.
A abordagem em cada um dos domínios anteriormente citados será pois a seguinte: (1) em primeiro lugar será lançada a importância da problemática; (2) de seguida apresentaremos a amostra, bem como os aspectos metodológicos da análise e finalmente, (3) abordaremos os principais resultados e seu significado.

\section{EXEMPLO 1}

\section{Epidemiologia da actividade física}

É grande a convicção, entre investigadores de diferentes quadrantes de interesses da Medicina e Ciências do Desporto, de que a actividade física diminui os factores de risco de um conjunto variado de condições mórbidas e parece possuir a capacidade de aumentar a longevidade (sobre esta matéria consultar 9,33,47). É também grande a convicção que um estilo de vida activo e saudável na vida adulta encontra as suas raízes na infância e adolescência. Daqui que a educação para a saúde tenda a percorrer toda a história de vida de cada um de nós e assente num conjunto de pressupostos dirigidos para a promoção da saúde em idades baixas. Tais pressupostos são apresentados por Kelder et al. (1994) do seguinte modo:

$1^{\circ}$ Um certo número de crianças tende a situar-se em zonas de risco fisiológico e comportamental.

$2^{\circ}$ Os riscos de natureza fisiológica tendem a evidenciar estabilidade (i.e., tracking moderado a substancial) da infância à idade adulta.

$3^{\circ} \mathrm{O}$ desenvolvimento de factores de risco fisiológico depende, largamente, das idades do seu aparecimento, sobretudo daquilo que se designa por comportamentos comprometedores da saúde (ex: dietas com baixo valor nutritivo; hábitos tabágicos; hábitos de inactividade física).

$4^{\circ} \mathrm{A}$ prevenção primária pode, e deve, ser realizada através da modificação de comportamentos que se pensa estarem associados com factores de risco fisiológico, antes que tais padrões comportamentais estejam profundamente enraizados e sejam mais resistentes à mudança. 
É a partir deste conjunto de pressupostos, e com base nas convicções da íntima associação da prática de actividade física e desportiva, que se tem produzido investigação sobre o tracking da actividade física. A título de exemplo, salientamos algumas pesquisas recentes realizadas por Glenmark et al. (1994) relativas à previsão dos níveis de actividade física de adultos, com base nos valores evidenciados pelos sujeitos durante a adolescência; por Pate et al. (1996) relativo ao tracking da actividade física de crianças num estudo com a duração de 3 anos; de Telama et al. (1996) também sobre o tracking da actividade física de crianças e adolescentes durante 3 anos num delineamento longitudinal-misto; e por Pate et al. (1999) acerca do tracking da actividade e inactividade física em crianças com 10 anos de idade.

A análise dos resultados destes estudos desagua, normalmente, de tabelas de contingência onde se calculam proporções de acordos ou o Kappa de Cohen, regressão múltipla, ou muito frequentemente em auto-correlações de Pearson ou Spearman. Apesar da relevância inegável destas pesquisas, pensamos que "sofrem" de algumas limitações do ponto de vista metodológico 26,28: (1) o significado substantivo a atribuir ao valor de $r$, assumindo uma ideia de causalidade que a noção de correlação nunca encerrou; (2) a limitação da amplitude dos dados nas medidas repetidas do mesmo indicador, reflectida na ausência de amostras aleatórias, ou no limite temporal da pesquisa; (3) o significado atribuído ao valor empírico de $r \geq 0.50$ para qualquer característica evidenciar estabilidade não ser, na quase totalidade dos casos, justificado; (4) a reduzida dimensão das amostras (normalmente $<100$ sujeitos), o que coloca sérios problemas na estimação de parâmetros, bem como na ausência de precisão da amplitude dos intervalos de confiança e generalização dos resultados; (5) a circunstância dos valores de $r$ não estarem corrigidos pelas estimativas de fiabilidade, implicando que os resultados mencionados na literatura sejam sempre sub-estimados, dado estarem contaminados por erros de medição.

Ora, é com base neste conjunto de "críticas", e a partir de inovações no plano metodológico e estatístico, que se propõe uma forma distinta para abordar o problema do tracking no domínio da actividade física.

\section{ASPECTOS METODOLÓGICOS Amostra}

A informação relativa a este estudo provém da famosa pesquisa (Leuven Growth Study) realizada na Universidade Católica de Lovaina sob a orientação do Prof. Gaston Beunen', em que 588 sujeitos do sexo masculino foram seguidos longitudinalmente durante 6 anos. No primeiro momento da observação, a idade média dos sujeitos era de 12.7 anos, e no último momento de registo era 17.7. À excepção de algumas medidas somáticas cujo registo era semestral, na maior parte dos outros indicadores as medições foram efectuadas anualmente. De um conjunto multifacetado de indicadores cobrindo aspectos do crescimento somático, maturação biológica, somatótipo, composição corporal, aptidão física e estatuto socio-económico, há também informação relativa à actividade física. Neste último domínio, a informação é muito detalhada. Contudo, para fins ilustrativos do modelo que a seguir se apresenta, focalizaremos a nossa atenção no indicador número de horas de prática desportiva formal, independentemente das horas de prática realizada nas aulas de Educação Física.

\section{Análise de estruturas de covariância}

O modelo de estruturas de covariância (MEC) é uma espécie de alquimia estatística moderna, dado considerar, numa formulação única altamente flexível e suficientemente genérica, as propostas de biometristas, econometristas e psicometristas (ver por exemplo em língua portuguesa, Maia, 1998a). Na sua essência, trata de conceber e realizar, numa abordagem única, a análise factorial e a regressão múltipla multivariada, com inclusão de variáveis latentes e variáveis observadas, com diferentes escalas de medida e sob diferentes tipos de distribuições. As unidades de análise não necessitam de ser medidas de forma contínua. Fortes desenvolvimentos metodológicos estão disponíveis para variáveis ordinais e dicotómicas. A MEC está fortemente teorizada (ver por exemplo Bollen, 1989; Joreskog, 1969; Joreskog

\footnotetext{
${ }^{1}$ Gostaríamos de expressar a nossa gratidão aos Profs. Doutores Gaston Beunen e Johan Lefevre pelo facto de nos facilitarem o acesso à sua base de dados.
} 
\& Sorbom, 1989; 1996), não cessa de ser alargada a outros horizontes analíticos (ver por exemplo Marcoulides \& Schumacker, 1996) e aplicada em diversos domínios que vão de estudos meramente descritivos a experimentais, percorrendo áreas tão distintas como são as ciências da educação e a epidemiologia, estudos econométricos ou de genética quantitativa.

O MEC é composto por dois sub-modelos, o de medida e o estrutural. O sub-modelo de medida, numa das suas formalizações mais simples e que nos interessa para ilustrar a noção de tracking será apresentado de seguida, a partir da operacionalização simples da noção complexa de actividade física. O modelo estrutural será ilustrado mais adiante. As preocupações dos peritos na avaliação das aptidões e/ou comportamentos reflectem-se, do ponto de vista operacional, na apresentação de baterias de testes ou instrumentos de trabalho mais simples, como são os questionários, que reflictam as noções de economia e parcimónia. Daqui que cada uma das componentes das aptidões ou de uma característica do comportamento do sujeito possa ser marcada por um único indicador. No caso presente, e a título de exemplo, a actividade física será imperfeitamente representada e marcada pelo indicador número de horas de prática desportiva. O modelo de medida, de acordo com a Teoria Clássica dos Testes, é pictograficamente representado do seguinte modo (ver Figura 1).

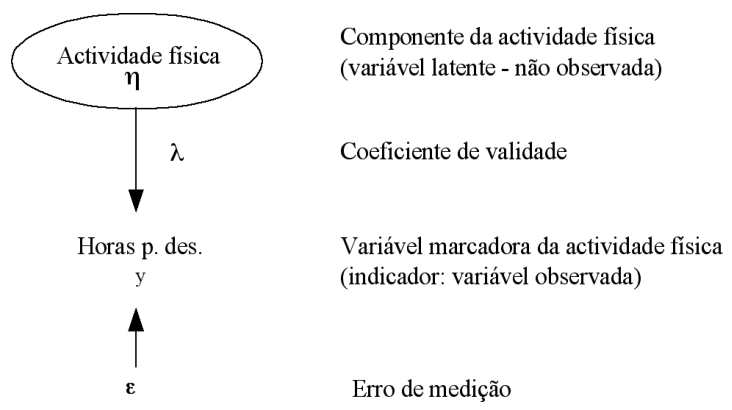

Figura 1. Especificação pictográfica para o modelo de medida da actividade física.

No contexto da MEC, as variáveis latentes ( $\eta$ ) são representadas em círculos ou elipses, e as variáveis observadas (y) em quadrados ou rectângulos. $\mathrm{O} \lambda$ refere-se, neste caso, ao valor de validade convergente (em termos de Análise Factorial é o loading estrutural) do indicador horas de prática desportiva para representar a noção de actividade física. $\mathrm{O} \varepsilon$ representa o erro de medição, a extensão de variância erro que reflecte o maior ou menor grau de fiabilidade dos resultados obtidos no questionamento das horas de prática desportiva.

O algoritmo que soluciona numericamente a MEC está disponível em software com diferentes parametrizações - o EQS (Bentler e Wu, 2001), o LISREL (Joreskog e Sorbom, 1996), e o Mplus (Muthén e Muthén, 2001). Para além destes três programas, há outros disponíveis no mercado e implementados em software estatístico bem conhecido - o COSAN (no SAS), o AMOS (no SPSS) e o SEPATH (no STATISTICA).

As estatísticas disponíveis nos resultados da aplicação destes programas para modelar estruturas de covariância são suficientemente esclarecedoras da sua vantagem relativamente ao simples cálculo de estatísticas descritivas como o r de Pearson ou de Spearman que a maior parte da pesquisa sobre o tracking disponibiliza. Destacamos, pois : (1) medidas de ajustamento global para a totalidade do modelo, considerando um equilíbrio entre complexidade e parcimónia; (2) estimativas pontuais e intervalares para os parâmetros fundamentais de cada modelo; (3) medidas de variância explicada; (4) estimativas de variância verdadeira não contaminada por erro de medição que se designam por coeficientes de tracking; (5) ajuda na localização de fontes de má especificação de cada um dos modelos.

O modelo que vamos descrever será apresentado de forma semântica, a que se seguirá a sua representação ou especificação pictográfica. Os resultados numéricos serão interpretados no contexto de cada formulação. Para não carregar o texto, não será referida a estrutura estatística de cada modelo. Os leitores interessados neste particular encontrarão nas referências matéria suficiente para uma viagem mais minuciosa a este território.

\section{0 modelo simplex de Markov: ideias genéricas. representação gráfica, hipóteses e resultados} Este modelo pretende descrever dois aspectos nucleares da dinâmica da mudança nos níveis de 
actividade física - sobretudo a noção de estabilidade de covariância (ou tracking) e a de mudança. A mudança é expressa pela magnitude da variação na componente aleatória $\left(\zeta_{\mathbf{i}}\right)$ e a estabilidade pela componente determinística $\left(\beta_{\mathrm{ji}}\right)$.

Assume-se que a continuidade ocorre quase que exclusivamente pelo efeito dos valores das variáveis em pontos diferidos do tempo, produzindo aquilo que se designa por modelo auto-regressivo de $1^{\mathrm{a}}$ ordem (Figura 2), dado que os valores em $t_{\mathbf{i}}$ são dependentes exclusivamente dos valores obtidos em $t_{\text {i-1. }}$. Não há pois lugar para efeitos não-adjacentes no tempo.

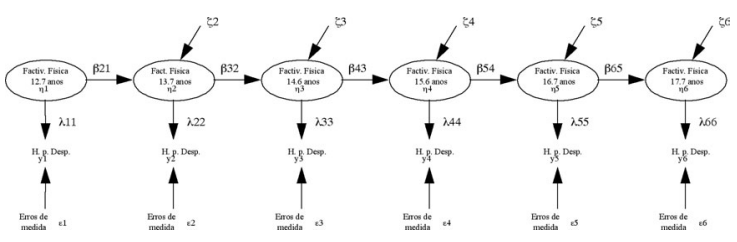

Figura 2. Especificação gráfica para o modelo simplex de Markov relativo à actividade física.

Nesta formulação, o valor da expressão da actividade física, por exemplo, em cada ponto do tempo é transferida para o ponto seguinte, fazendo do valor em $t_{\mathbf{i}}$ formalmente dependente do valor em $t_{\mathbf{i}-1}$. Isto significa que o valor da actividade física aos 12.7 anos influencia directamente, é o preditor único, do valor aos 13.7 anos, o dos 13.7 anos é preditor único do valor aos 14.6 anos e assim sucessivamente. Trata-se de um modelo auto-regressivo ordenado no tempo face à estrutura espacial mais ou menos fixa das ocasiões de avaliação. Este modelo é também designado de Markov (nome do eminente estatístico russo que formulou a estacionaridade em sistemas desta natureza), sendo possível separar a estabilidade $\left(\beta_{\mathbf{j i}}=\right.$ coeficiente de regressão) da distribuição dos valores dos sujeitos, da instabilidade da sua posição relativa no tempo $\left(\zeta_{\mathbf{i}}=\right.$ instabilidade na variância, também designada por perturbação na estacionaridade do sistema). A magnitude do valor de $\zeta_{\mathbf{i}}$ reflecte, pois, os efeitos aleatórios de mudança ocorrida em $t_{\mathbf{i}}$, por outros factores que não os implicados por $t_{\mathbf{i}-1}$.
Por questões de identificação de modelos desta natureza, a variância da variável latente (i.e. a actividade física) está expressa nas unidades de medida do indicador considerado - horas de prática desportiva (para tal fixam-se os valores de $\lambda_{\text {ii }}$ em 1). Tal como foi anteriormente referido, os valores de $\varepsilon_{\mathbf{i}}$ representam erros de medição, e os $\lambda_{\text {ii }}$ são indicadores de validade convergente.

Este tipo de modelos foram solucionados matematicamente por Joreskog (1970) no contexto da MEC, têm sido utilizados para estudar o crescimento académico (Werts, Linn \& Joreskog, 1977), processos econométricos (Baltagi, 1995), sociológicos (Finkel, 1995), estruturas hierárquicas de aprendizagem (Hill, 1987), dados de natureza educacional (Cuttance \& Ecob, 1987), bem como em aplicações desenvolvimentalistas (McArdle \& Aber, 1990). O seu uso em estudos de natureza epidemiológica da aptidão física e em desenvolvimento motor deve-se a Maia et al. (1998a; 1998b; 2001).

O Quadro 1 apresenta a matriz de auto-correlação relativa às horas de prática desportiva nos seis pontos do tempo. Estes valores de auto-correlação são normalmente designados por valores de tracking da actividade física. Na sua generalidade, a magnitude dos resultados é baixa, sugerindo a inexistência de tracking.

Quadro 1. Matriz de auto-correlação para os valores relativos às horas de prática desportiva.

\begin{tabular}{lllllll} 
Idade & 12.7 & 13.6 & 14.6 & 15.6 & 16.7 & 17.7 \\
\hline 12.7 & 1.00 & & & & & \\
13.7 & 0.45 & 1.00 & & & & \\
14.6 & 0.39 & 0.52 & 1.00 & & & \\
15.6 & 0.31 & 0.35 & 0.52 & 1.00 & & \\
16.7 & 0.28 & 0.33 & 0.42 & 0.54 & 1.00 & \\
17.7 & 0.25 & 0.28 & 0.37 & 0.45 & 0.58 & 1.00
\end{tabular}

Uma leitura mais atenta desta matriz revela dois aspectos importantes: (1) os valores de correlação vão diminuindo à medida que nos afastamos da diagonal principal, i.e, em cada coluna, e (2) aumentam ao longo de cada linha. Tal estrutura tem propriedades matemáticas muito importantes, sendo um caso 
particular do simplex de Guttman, e que foram solucionadas por Joreskog (1970). Dado que os valores de correlação se referem aos resultados obtidos das respostas relativamente ao número de horas de prática desportiva e possuem uma quantidade de erro de medição, esta matriz é designada de quasi-simplex, uma vez que não se verifica a seguinte igualdade,

$$
r_{i j}=\left(r_{i, i+1}\right)\left(r_{i+1,1+2}\right) \ldots\left(r_{j-1, j}\right) \text {, para } i<j
$$
ou seja, num perfeito simplex, a correlação entre duas variáveis i e j seria igual ao produto das correlações entre todos os pares adjacentes.

Dado que no modelo quasi-simplex se analisa exclusivamente a estrutura da covariância dos valores obtidos, a hipótese que aqui vai ser colocada é a seguinte:

Tal como quantificada pelo número de horas de prática desportiva, a actividade física evidencia uma forte estabilidade de covariância no tempo. A mudança ocorrida implica que os sujeitos mantenham as suas posições relativas dos 12.7 aos 17.7 anos de idade. Ou seja, face à estabilidade interindividual das trajectórias de crescimento nos níveis de actividade física, é relativamente fácil predizer valores futuros de actividade física dos sujeitos a partir do conhecimento de valores prévios. Dito de um outro modo, pode dizer-se que a forte estabilidade significa uma canalização acentuada na trajectória de cada sujeito. Se a instabilidade na actividade física fosse reduzida, então estaria aberto o caminho para a previsão de valores futuros de actividade física, dada a presença de um tracking elevado.

Dada a enorme flexibilidade da MEC, foram testados três modelos alternativos:

- Um primeiro modelo sem qualquer restrição nos parâmetros $\left(\beta_{\mathbf{i j}}\right.$, $\zeta_{\mathbf{i}}$ e $\left.\varepsilon_{\mathbf{i}}\right)$, à excepção de necessidade de identificação da solução para se conseguir convergência do algoritmo - $\varepsilon_{1}$ e $\varepsilon_{2}$ serem iguais, e o mesmo ocorre para $\varepsilon_{5}$ e $\varepsilon_{6}$ em zero (ver Joreskog, 1970). Este modelo é normalmente considerado de baseline, dado que permite testar de forma hierárquica um outro conjunto de modelos que podem ser mais parcimoniosos. Este é o modelo que representa a hipótese previamente formulada.
- O segundo modelo apresenta restrições nos parâmetros $\beta_{\mathbf{i j}}$ e $\varepsilon_{\mathbf{i}}$. A primeira refere-se à sugestão de uma estabilidade de covariância perfeita para a actividade física, também conhecida por estabilidade paralela; a segunda propõe o mesmo valor para a variância erro. Isto quer dizer que os valores de fiabilidade das respostas dos sujeitos nos diferentes pontos do tempo são os mesmos - uma hipótese altamente plausível.

- O terceiro modelo considerou a possibilidade de não haver tracking - a covariância entre medidas repetidas no tempo não seria significativamente diferente de zero. Daqui que só houvesse variâncias independentes. Este é o modelo mais restritivo.

Um modelo descritor perfeito da estrutura de covariância amostral teria 0 graus de liberdade e $\chi^{2}=0$. Neste caso, o modelo representaria a realidade do fenómeno em estudo, o que é, em si mesmo, uma impossibilidade, dado que, por definição, um modelo é uma aproximação da representação da realidade. Contudo, mostra desde logo que um bom modelo, entre outros aspectos da análise, será aquele que possuir um menor número de graus de liberdade e um valor mais reduzido de $\chi^{2}$ - encontro entre parcimónia e complexidade de representação e explicação da matriz de covariância.

Todos os cálculos foram realizados no programa Mplus (Muthén e Muthén, 2001), recorrendo ao método robusto de estimação por máxima verosimilhança. As principais estatísticas descritivas dos dados encontram-se no Quadro 2.

Quadro 2. Mediana (Me), média (M), desvio padrão (Dp), assimetria (G1) e achatamento (G2) das horas de prática desportiva nos seis momentos de registo.

\begin{tabular}{llllll} 
Anos & Me & M & Dp & G1 & G2 \\
\hline 12.7 & 2.33 & 3.33 & 3.34 & 1.64 & 3.63 \\
13.6 & 2.83 & 3.64 & 3.37 & 1.41 & 2.15 \\
14.6 & 3.33 & 4.08 & 3.33 & 1.50 & 2.86 \\
15.7 & 4.00 & 4.88 & 4.01 & 1.49 & 2.73 \\
16.7 & 4.33 & 5.10 & 3.97 & 1.30 & 1.88 \\
17.7 & 3.88 & 4.86 & 4.07 & 1.19 & 1.23
\end{tabular}


É evidente a forte assimetria e achatamento das distribuições da variável em estudo ao longo dos seis anos. Quer os testes univariados à assimetria, quer ao achatamento produziram resultados altamente significativos $(\mathrm{p}<0.01)$. Tal traduz uma violação à normalidade das distribuições. Daqui que, qualquer esforço de modelação da estrutura dos dados exija procedimentos robustos.

Os principais resultados do ajustamento dos modelos testados encontram-se no Quadro 3.

Quadro 3. Estatísticas de ajustamento global dos modelos previamente apresentados.

Modelos

$\begin{array}{lllll}\chi^{2} & \mathrm{gl}^{1} & \mathrm{p}^{2} & \mathrm{CFI}^{3} & \mathrm{RMSEA}^{4}\end{array}$

Modelo baseline

$\begin{array}{lllll}3.13 & 6 & 0.79 & 1.00 & 0.00\end{array}$

$\begin{array}{lllllll}\text { Modelo de tracking perfeito } \quad 21.02 & 11 & 0.03 & 0.98 & 0.04\end{array}$

Modelo de tracking perfeito,

$\begin{array}{llllll}\text { com variância erro constante } & 29.26 & 14 & 0.00 & 0.98 & 0.05\end{array}$

Modelo de ausência de tracking $\begin{array}{lllll}659.64 & 15 & 0.00 & \text { - }\end{array}$

1=graus de liberdade; $2=v a l o r$ da prova; $3=$ Comparative Fit Index; $4=$ Root Mean Square Error of Approximation

Os resultados do ajustamento quer global quer local sugerem a forte plausibilidade do primeiro modelo face ao baixo valor do Qui-quadrado, e sobretudo à circunstância do valor da prova ser de 0.79. Ao não ser rejeitada a hipótese nula, que é a hipótese previamente lançada está encontrado o melhor modelo em contraste com os outros que rejeitam sempre as suas hipóteses nulas (detalhes de contraste estatístico entre modelos é encontrado, por exemplo, em Foguet e Gellart, 2000; Maia, 1998a; Raykov e Marcoulides, 2000). Os aspectos mais relevantes da melhor solução estão no Quadro 4.
Quadro 4. Solução do modelo simplex de Markov para a actividade física marcada pelo número de horas de prática desportiva.

Actividade Física

\begin{tabular}{ll}
\hline Coeficiente de estabilidade $(\beta \mathrm{ji})$ & \\
$12.7-13.7$ & 0.78 \\
$13.7-14.6$ & 0.81 \\
$14.6-15.6$ & 0.77 \\
$15.6-16.7$ & 0.82 \\
$16.7-17.7$ & 0.84 \\
Variância explicada $\left(\mathrm{R}^{2}\right)$ & \\
$12.7-13.7$ & 0.60 \\
$13.7-14.6$ & 0.65 \\
$14.6-15.6$ & 0.60 \\
$15.6-16.7$ & 0.66 \\
$16.7-17.7$ & 0.70 \\
Instabilidade na variância $\left(\zeta_{\mathrm{i}}\right)$ & \\
12.7-13.7 & 0.40 \\
$13.7-14.6$ & 0.35 \\
$14.6-15.6$ & 0.40 \\
15.6-16.7 & 0.34 \\
16.7-17.7 & \\
Medidas de ajustamento global & \\
Qui-Quadrado & 0.30 \\
CFI & 3.13 \\
RMSEA & 1.00 \\
& 0.00
\end{tabular}

Os valores dos coeficientes de estabilidade (valores estandardizados) são moderados a elevados, expressão visível da manutenção da posição relativa de cada sujeito na sua trajectória de crescimento da actividade física em relação aos outros. Os valores de variância de cada equação estrutural são moderados, situando-se entre os $60 \%$ e os $70 \%$. Daqui se infere que a instabilidade das trajectórias seja baixa, contudo presente.

Os resultados da modelação revelam uma canalização moderada a elevada de desenvolvimento interindividual nos níveis de actividade física. Conforme é visível no Quadro 4, a instabilidade das medidas adjacentes no tempo é baixa e situa-se, quase sempre na mesma magnitude, entre 0.30 e 0.40 . Se porventura os valores de estabilidade fossem reduzidos, o que não se verifica neste estudo, poderíamos estar na presença de: 
- Fiabilidade e validade convergente baixas para a variável marcadora da actividade física. Tal não parece ser o caso.

- Saltos mais ou menos aleatórios no processo de crescimento da actividade física, ou eventualmente a circunstância da mudança se processar por estádios. Ora nem uma nem outra possibilidades parecem estar patentes nos resultados.

Um aspecto muito relevante do modelo simplex resulta no cálculo de valores de actividade física não contaminados por erros de medição. A matriz de tais valores, designada por matriz de auto-correlação ou de tracking deste processo, está no Quadro 5. É evidente uma forte diferença destes resultados relativamente aos que se encontram no Quadro 1. Os valores de tracking estimados a partir do modelo simplex são moderados, em nítido contraste com os primeiros que sugeriam a possibilidade do tracking da actividade física ser baixo a irrelevante.

Quadro 5. Matriz de auto-correlação ou tracking da actividade física [estimação a partir do modelo simplex].

\begin{tabular}{lllllll} 
Idade & 12.7 & 13.6 & 14.6 & 15.6 & 16.7 & 17.7 \\
\hline 12.7 & 1.00 & & & & & \\
13.7 & 0.78 & 1.00 & & & & \\
14.6 & 0.63 & 0.81 & 1.00 & & & \\
15.6 & 0.48 & 0.62 & 0.77 & 1.00 & & \\
16.7 & 0.39 & 0.51 & 0.63 & 0.82 & 1.00 & \\
17.7 & 0.33 & 0.43 & 0.53 & 0.68 & 0.84 & 1.00
\end{tabular}

Em suma, são aqui salientadas duas ideias fundamentais. A primeira radica na enorme riqueza conceptual e analítica do recurso à MEC para estudar o tracking, face não só à possibilidade em postular hipóteses rivais para os mesmos dados, mas também à disponibilidade do software em fornecer uma variedade substancial de estatísticas que não se encontram nos estudos anteriores sobre o tracking que recorrem quase que exclusivamente a correlações de Pearson ou Spearman. A segunda ideia mostra a dependência estrutural moderada e elevada dos níveis de actividade física em cada ano face ao anterior, e o mesmo ocorre para os valores do tracking- moderados a elevados. Contudo, não deixa de ser interessante verificar que os resultados da instabilidade se situam entre 0.30 e 0.40 , o que mostra alguma labilidade neste comportamento ao longo dos anos.

\section{EXEMPLO 2}

\section{Performance desportivo-motora}

Um dos aspectos nucleares de todo o processo de treino estruturado e planeado na direcção do alto rendimento é o da preocupação com o comportamento da dinâmica da performance. Contudo, e inexplicavelmente, não parece ser muito evidente uma forte preocupação dos investigadores por esta temática $^{2}$, muito menos dos treinadores, a "julgar" pela ausência de estudos ou reflexões escritas sobre a matéria. Por exemplo, manuais recentes sobre o treino desportivo (Bompa, 1999; Siff \& Verkhoshansky, 2000), ou sobre o atletismo ${ }^{3}$ não relevam qualquer apetência pelo estudo da dinâmica da performance em função do tempo. Do mesmo modo, revistas da actualidade (ver por exemplo a "Revue AEFA", a "Track Coach" ou a "Atletismo Español") também evidenciam algum silêncio sobre tal matéria. Muito mais pobre é tal produção em Portugal, onde não se vislumbra, até ao momento, qualquer tentativa de modelação do dinamismo da performance desportivo-motora em função do tempo ${ }^{4}$. Contudo, não deixamos de salientar o alvo de todo o processo de treino com vista ao alto rendimento - o sucesso competitivo. Não duvidamos que o problema do dinamismo da performance seja motivo de forte preocupação de qualquer treinador. Infelizmente, as suas reflexões raramente tomam forma de letra publicada em revistas da especialidade.

\footnotetext{
${ }^{2}$ Uma consulta à base de dados da Sport Discus não produziu resultados satisfatórios dada a escassez de produção na matéria da modelação da dinâmica da performance em função do tempo. Foram usadas diversas combinações de palavras-chave: modeling, performance, long-jump, track and field, dynamics.

${ }^{3}$ Uma consulta na bibliografia disponível na biblioteca da FCDEF-UP não revelou qualquer resultado frutífero nesta matéria específica.

${ }^{4}$ Não conhecemos qualquer publicação sobre esta matéria que tenha analisado dados longitudinais de atletas ou de equipas.
} 
A título de exemplo ilustrativo do tracking, servir-nos-emos de informação longitudinal proveniente de uma especialidade marcada pela expressão da força explosiva - o salto em comprimento.

\section{ASPECTOS METODOLÓGICOS}

\section{Amostra}

Os dados para este estudo provêm das melhores marcas anuais de doze saltadores em comprimento portugueses. Isto significa que possuímos informação completa sobre todos os saltadores (ver Quadro 6).

Quadro 6. Valores da melhor marca no salto em comprimento de 12 atletas durante 6 anos (os resultados estão em metros).

\begin{tabular}{lcccccc} 
Atletas & $\mathbf{1}$ & $\mathbf{2}$ & $\mathbf{3}$ & $\mathbf{4}$ & $\mathbf{5}$ & $\mathbf{6}$ \\
\hline 1 & 6.23 & 6.64 & 7.27 & 7.33 & 7.55 & 7.45 \\
2 & 6.50 & 6.59 & 7.00 & 7.24 & 7.18 & 7.46 \\
3 & 6.21 & 6.59 & 7.00 & 7.28 & 7.71 & 7.52 \\
4 & 6.85 & 7.15 & 7.37 & 7.30 & 7.40 & 7.32 \\
5 & 6.65 & 6.94 & 7.10 & 7.05 & 7.02 & 6.78 \\
6 & 6.75 & 6.94 & 6.86 & 6.89 & 6.73 & 7.33 \\
7 & 6.57 & 6.75 & 6.88 & 7.00 & 6.69 & 6.91 \\
8 & 6.49 & 6.73 & 6.80 & 6.57 & 7.17 & 6.85 \\
9 & 6.65 & 6.66 & 6.81 & 6.61 & 7.25 & 6.98 \\
10 & 6.78 & 6.66 & 6.85 & 6.77 & 6.92 & 6.68 \\
11 & 6.36 & 6.78 & 6.83 & 6.93 & 7.16 & 7.05 \\
12 & 6.52 & 6.65 & 6.77 & 6.69 & 6.71 & 6.88 \\
Média & 6.64 & 6.77 & 6.97 & 6.97 & 7.15 & 7.10
\end{tabular}

\section{PROCEDIMENTOS E PRINCIPAIS RESULTADOS}

Uma forma demasiadamente elementar de análise dos resultados seria, muito simplesmente, a centração da atenção nos valores médios. Tal atitude parece-nos superficial e insuficiente, porque não explora a totalidade das características da informação individual disponível.

A título de mero exemplo, vejamos o comportamento dos resultados de dois atletas (ver Figura 3), contrariando fortemente a tirania dos valores médios. Os gráficos revelam uma diversidade inequívoca de comportamentos da performance dos dois sujeitos que jamais seria capturada pela simples apreciação dos valores médios.
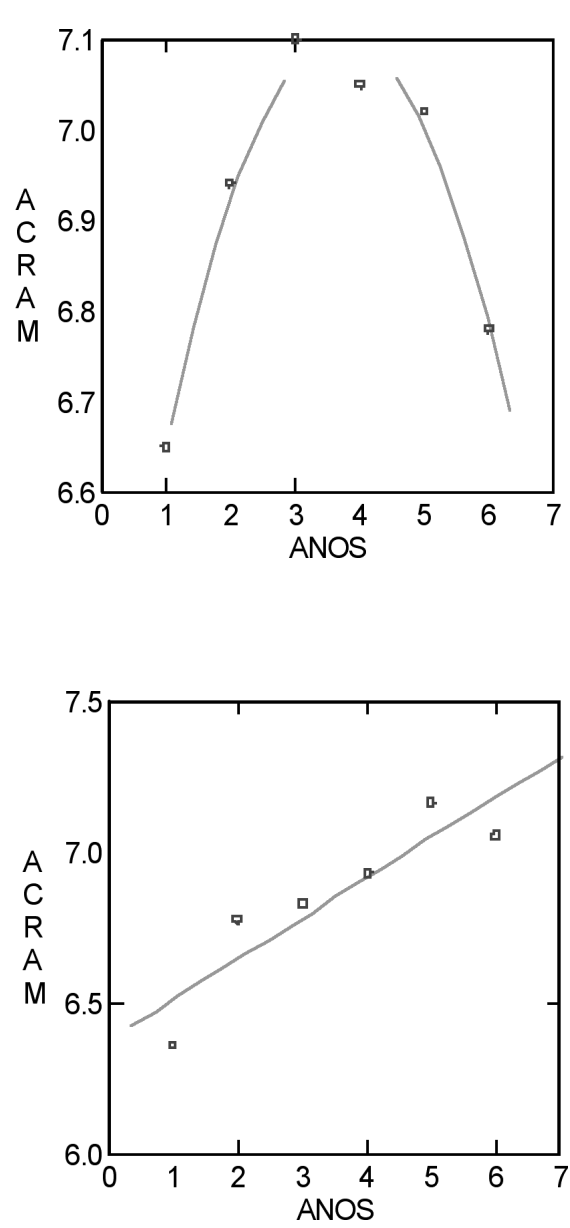

Figura 3. Representação do ajustamento de
modelos distintos de regressão onde se nota
um comportamento não-linear dos resultados
$\left(R^{2}=0.98\right]$ no painel de cima e linear $\left(R^{2}=0.81\right]$
no de baixo.

Um conceito extremamente interessante que pode ser aplicado ao estudo da performance dos saltadores é o de canalização. Este conceito foi inicialmente proposto em 1957 pelo geneticista inglês Waddington no seu livro Strategy of the Genes, uma reflexão teórica sobre aspectos da Biologia, lançando-se na aventura concreta da Biologia do Desenvolvimento a partir de informação proveniente 
da embriologia e da genética. O autor sugeria, no contexto biológico, que o desenvolvimento ou o crescimento se processava ao longo de um dado canal numa paisagem epigenética com múltiplas possibilidades, onde era evidente não só um comportamento regular do fenómeno, como também a tendência para se dirigir para um alvo geneticamente determinado e actualizado pelo envolvimento.

Tanner (1978) estende esta noção ao processo de crescimento somático, e Baumgartner e Roche (1988) e Roche e Baumgartner (1988) utilizaram-no para pesquisar as alterações na distribuição do padrão de gordura corporal durante o crescimento do ser humano.

A noção de canalização emerge de forma clara quando se interpretam valores repetidos do crescimento somático de um dado sujeito numa carta de crescimento, sobretudo quando se monitoriza o seu processo e se pretende situá-lo no contexto de referências locais ou internacionais.

Sempre que as medidas repetidas no tempo de uma variável (por exemplo a altura, o peso, ou um indicador relevante da performance desportivo-motora) de um dado sujeito permanecem entre um par de percentis adjacentes (por exemplo $\mathrm{P}_{10}-\mathrm{P}_{25} ; \mathrm{P}_{50}-\mathrm{P}_{75}$ ), ou que não se desviam mais do que um dado centil maior ou para o canal contíguo, diz-se que se está na presença de canalização. De acordo com

Baumgartner e Roche (1988) e Roche e Baumgartner (1988), este conceito é credor de uma forte aplicação na pesquisa sobre o crescimento e desenvolvimento somático e motor ou da performance desportiva intra-individual, dado implicar um prognóstico relativamente ao valor a ser atingido no estado adulto, ou no nível mais elevado de performance. Daqui que esta noção exija alguma modelação matemática para dados de comportamento linear ou não-linear.

Estamos na presença de mudança de canal de desenvolvimento ou da performance desportiva sempre que a alteração das medidas repetidas no tempo implique uma transição para canais não adjacentes, por exemplo passar do canal $\mathrm{P}_{10}-\mathrm{P}_{25}$ para o canal $\mathrm{P}_{50}-\mathrm{P}_{75}$. Pode acontecer que por motivos de "forte conspiração" do envolvimento (por exemplo sub-nutrição, condições desfavoráveis de treino, alteração substancial das condições da competição, ou do próprio sujeito) haja um desvio do canal de desenvolvimento ou da performance. Se se remover este efeito nocivo, o organismo possui uma tendência inata para voltar ao seu canal original. Este fenómeno designa-se por recanalização.

A aplicação destas noções tem sido extremamente fértil na descrição e interpretação do crescimento somático (ver por exemplo Park et al., 1997; Tanner, 1978).

Um modo altamente promissor de análise e interpretação dos resultados apresentados no Quadro 6 é o recurso à segunda corrente de pensamento metodológico, no que ao tracking diz respeito, se nos "adaptarmos" aos conteúdos provenientes da ideia de canalização. Para tal faremos uso da sua implementação no software estatístico Longitudinal Data Analysis (Schneiderman e Kowalski, 1993), recorrendo exclusivamente ao Kappa de Cohen e ao gama de Foulkes e Davies (sobre esta matéria ver Kowalski e Schneiderman, 1992).

\section{O Kappa (K) de Cohen}

Este procedimento estipula a existência de tracking (i.e., estabilidade ${ }^{5}$ na performance) se os sujeitos tenderem a permanecer no mesmo quantil (track ou canal) da distribuição. A pergunta chave é pois: quantas vezes é que a marca de cada atleta está no seu quantil, que é calculado em função dos valores das marcas dos atletas em cada ponto do tempo. Este procedimento não exige qualquer a priori acerca da forma da curva de crescimento da performance, bem como não reclama qualquer avaliação da normalidade da distribuição, dado tratar-se de uma estatística não-paramétrica. Baseado nas sugestões de Landis e Koch (1977), considera-se a seguinte interpretação para os valores do Kappa: $\mathrm{K} \geq 0.75=$ excelente; $\mathrm{K} \geq 0.40$ e $\mathrm{K}<0.75=$ moderado a bom; $\mathrm{K}<0.40=$ mau.
${ }^{5}$ Por estabilidade não se entende a ausência de mudança, a não ser que o valor das médias, ou os valores de cada sujeitos não se alterem substancialmente no tempo. O conceito de estabilidade é bem dinâmico e plural, podendo evidenciar características que a qualificam de paralela, estrita ou monotónica (sobre esta matéria ver Maiaet al., 1998a; Maia et al., 2001). 
A Figura 4 mostra, para a distribuição em causa, quatro canais que pretendem descrever a performance no tempo das sub-categorias de atletas da amostra, e o Quadro 7 contém os valores dos percentis, bem como os respectivos pontos de corte das marcas. Os canais podem ser considerados como zonas distintas de performance de sub-grupos de saltadores.

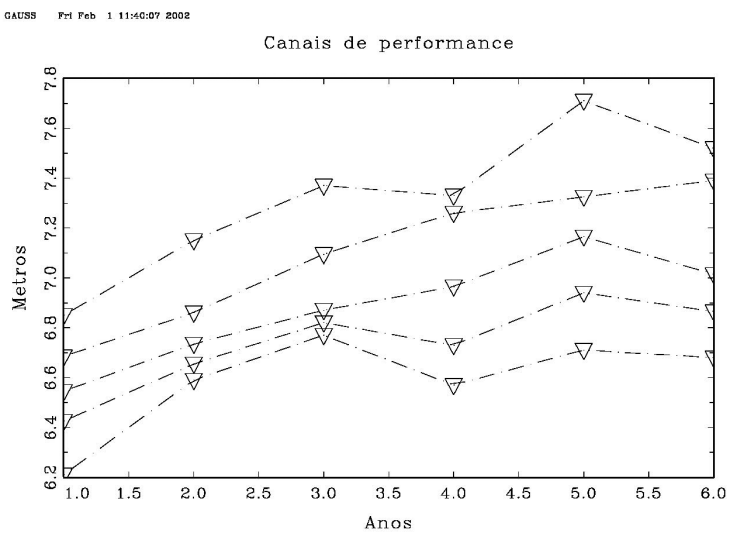

Figura 4. Canais da distribuição dos valores da performance dos atletas em função do tempo.

Quadro 7. Pontos de corte dos principais percentis nos seis momentos.

\begin{tabular}{lcccccc} 
Centis & $\mathbf{1}$ & $\mathbf{2}$ & $\mathbf{3}$ & $\mathbf{4}$ & $\mathbf{5}$ & $\mathbf{6}$ \\
\hline P100 & 6.85 & 7.15 & 7.37 & 7.33 & 7.71 & 7.52 \\
P75 & 6.67 & 6.86 & 7.10 & 7.26 & 7.33 & 7.39 \\
P50 & 6.55 & 6.74 & 6.87 & 6.97 & 7.17 & 7.02 \\
P25 & 6.43 & 6.66 & 6.82 & 6.73 & 6.94 & 6.87
\end{tabular}

Na Figura 5 estão incluídos os canais da distribuição quantílica dos resultados, bem como os traçados da performance de cada atleta.

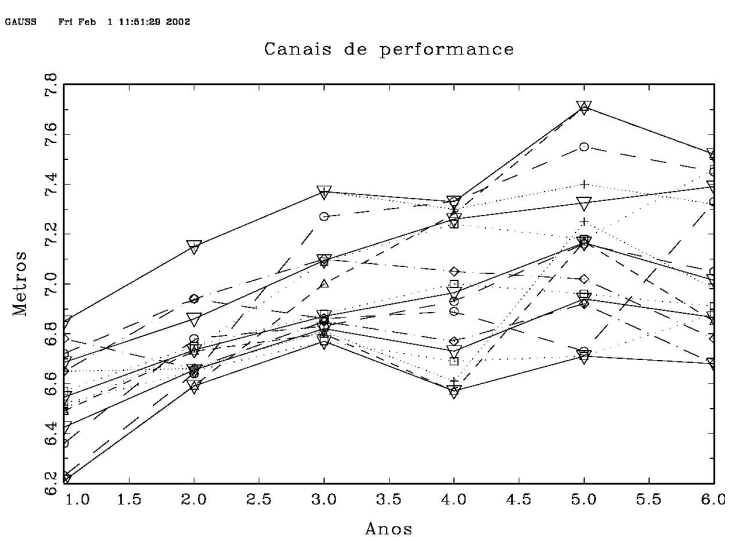

Figura 5. Canais de performance e trajectórias individuais não modeladas.

Os resultados da estabilidade da performance de cada atleta estão no Quadro 8.

Quadro 8. Valor de Kappa para cada atleta, bem como a frequência de permanência no respectivo canal.

\begin{tabular}{lllll} 
Kappa individual & Canal 1 & Canal 2 & Canal 3 & Canal 4 \\
\hline 0.47 & 2 & 0 & 0 & 4 \\
0.40 & 0 & 1 & 4 & 1 \\
0.27 & 2 & 0 & 1 & 3 \\
0.67 & 0 & 0 & 1 & 5 \\
0.13 & 1 & 1 & 2 & 2 \\
0.13 & 1 & 2 & 1 & 2 \\
0.47 & 0 & 2 & 4 & 0 \\
0.26 & 3 & 2 & 1 & 0 \\
0.20 & 2 & 2 & 2 & 0 \\
0.27 & 2 & 3 & 0 & 1 \\
0.27 & 1 & 3 & 2 & 0 \\
0.47 & 4 & 2 & 0 & 0
\end{tabular}

Conforme é evidente do Quadro anterior, o valor da estatística Kappa de cada sujeito é baixo, à excepção do atleta número 4, cujo Kappa é de 0.67 , salientando o facto de estar 5 vezes no seu quantil ou canal de performance mais elevado, e só uma vez no canal imediatamente inferior. Contudo, é importante considerar a dinâmica dos seus resultados (ver Quadro 6). Ainda que se situe no canal mais elevado de per- 
formance, e a maior frequência de resultados esteja no canal 4, as cinco últimas marcas salientam um carácter algo "ondulatório - de 7.15 passa para 7.37, depois desce para 7.30, sobe para 7.40, e volta a descer para 7.32. O sujeito número 6 , pelo contrário, possui um Kappa baixíssimo $(K=0.13)$. Está uma vez no canal 1, duas no canal 2 , uma no canal 3 e duas no canal 4. Se esta fosse a ordem da sua performance, i.e. a mudança sistemática para canais superiores de performance, o valor do tracking, ainda que baixo, traduziria os incrementos sistemáticos dos resultados deste atleta. Contudo, este não é o seu comportamento. Basta para tanto apreciar as suas marcas no Quadro 6.

O valor do Kappa para a totalidade dos sujeitos é de $0.11 \pm 0.04$, e os respectivos limites de confiança para $95 \%$ são $0.03 ; 0.20$. Estes resultados sugerem a ausência de estabilidade na performance dos saltadores. Tal significa, no contexto do próprio treino, um comportamento inconsistente nas melhores marcas de cada ano, o que reduz muito substancialmente qualquer aventura de previsão para o grupo e para cada atleta em particular.

\section{0 gama $(\gamma)$ de Foulkes e Davies}

Este procedimento pretende examinar a quantidade de indivíduos que mantêm a mesma posição relativa (rank relativo). Isto é, calcula a probabilidade de duas curvas ou perfis aleatórios de performance não se entrecruzarem. O tracking será perfeito quando o grupo de perfis de crescimento individual da performance não se entrecruzarem, i.e. quando a sua posição relativa no seio da distribuição for mantida no tempo do estudo.

Se o valor de $\gamma=0.50$ não se verifica qualquer tracking; se $\gamma=1.00$, o tracking é perfeito, e se $\gamma<0.50$, o tracking é irrelevante.

Dado que o $\gamma$ de Foulkes e Davies é uma estatística não-paramétrica, não há qualquer pressuposto a cumprir acerca da forma da curva de crescimento da performance. É evidente que quanto mais simples for a curva, tanto mais elevado será o valor de $\gamma$.

Os valores da performance dos atletas foram submetidos, sequencialmente, a um ajustamento linear e depois a um quadrático que se revelou mais adequado $(F=3.50, p=0.06)$, e que se encontram representados, após modelação, na Figura 6.

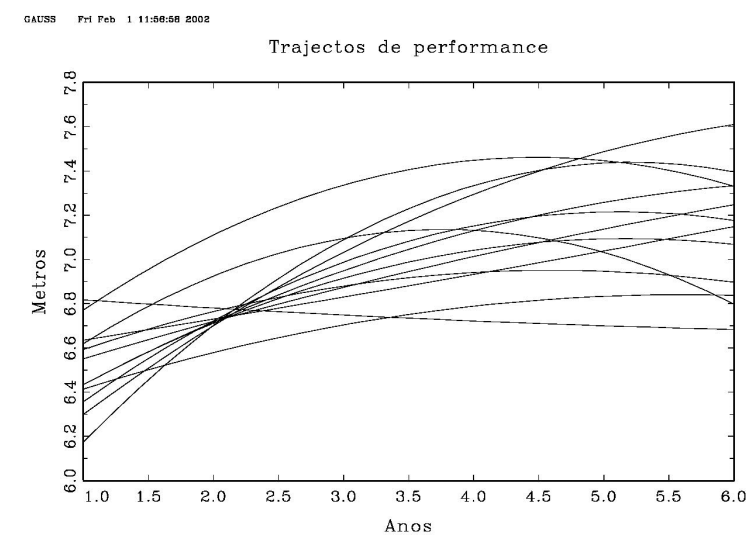

Figura 6. Modelação não linear dos trajectos individuais da performance.

Os traçados da performance do salto representados na figura anterior, modelados por uma função polinomial de grau 2, mostram o forte cruzamento dos perfis da performance dos sujeitos.

$\mathrm{O} \gamma$ de Foulkes e Davies evidenciou um valor de $0.19 \pm 0.06$, cujos limites de confiança a $95 \%$ são $0.08 ; 0.32$. De acordo com as sugestões anteriores para os valores de $\gamma$, estamos na presença de tracking irrelevante, ou dito de um modo mais adequado, na ausência de tracking ou canalização da performance dos atletas.

Em suma, é nossa convicção a forte utilidade do conceito de canalização da performance, uma faceta do tracking, quando se estuda a dinâmica da performance. O recurso aos procedimentos estatísticos e gráficos anteriores e aquilo que sugerem, torna tal matéria da máxima importância para o analista da performance e para o treinador, dado apreciarem de modo distinto os resultados do treino sistemático e planeado a que se submete os atletas. Esta é uma forma interessante de monitorizar, em curto, médio ou longo prazo, a dinâmica de resposta dos atletas ao treino e à competição.

Esperamos que estes exemplos simples de aplicação da ideia do tracking tenham sido suficientemente esclarecedores da sua importância quando lidamos com dados longitudinais, e procuramos perceber aspectos da sua dinâmica interna. O modelo simplex de Markov e as sugestões de Cohen ou Foulkes e 
Davies são auxiliares preciosos no entendimento da estabilidade e mudanças individuais e do grupo.

Contudo, é importante salientar que modelos mais sofisticados podem responder a questões bem mais complexas no domínio dos delineamentos longitudinais, como sejam, a título de mero exemplo: (1) a da determinação do grau de influência de preditores, invariantes ou não no tempo, na dinâmica dos registos longitudinais; (2) a da identificação da influência recíproca de dois processos dinâmicos de desenvolvimento da própria performance em pontos equidistantes do tempo, ou estão desfasados temporalmente; (3) a possibilidade em lidar com sub-grupos ou classes distintas de sujeitos cujo dinamismo da mudança é diferente e cujos preditores actuam de modo diverso nos sub-grupos de sujeitos; (4) ou ainda a possibilidade em lidar com informação omissa numa tabela de dados longitudinais, a eventualidade de efectuar previsões da performance, ou a classificação de atletas em distintos canais ou níveis de rendimento. Este tipo de questões e a sua modelação têm sido aplicados com sucesso noutras áreas do conhecimento de cariz desenvolvimentalista, i.e., em que processos de dinamismo longitudinal estão presentes. Só esperam que alguém os traga para o domínio das Ciências do Desporto. O ganho descritivo e interpretativo será enorme. Aguardemos atentamente o futuro de tais aventuras.

\section{CORRESPONDÊNCIA}

José António Ribeiro Maia

Laboratório de Cineantropometria

e Estatística Aplicada

Faculdade de Ciências do Desporto

e de Educação Física

Rua Dr. Plácido Costa, 91

4200.450 Porto

Portugal

jmaia@fcdef.up.pt 


\section{BIBLIOGRAFIA}

1. Andersen L B (1996). Tracking of risk factors for coronary heart disease from adolescence to young adulthood with special emphasis on physical activity and fitness.

Dissertação de doutoramento. University of Kopenhagen, Danish State Institute of Physical Education: Copenhagen.

2. Baltagi BH (1995). Econometric Analysis of Panel Data. John Wiley: Chichester.

3. Baumgartner RN, Roche AF (1988). Tracking of fat pattern indices in childhood: the Melbourne Growth Study. Human Biology. 4:549-567.

4. Bentler PM, Wu E (2001). EQS 6.0 (Beta version). Multivariate Software, Inc: Encino.

5. Bijleveld CCJH, van der Kamp LJT, Mooijart A, van der kloot WA, van der leeden R, and van der Burg E (1998). Longitudinal Data Analysis. Designs, Models and Method. Thousand Oaks: Sage Publications.

6. Bloom BS (1964). Stability and Change in Human Characteristics. New York: John Wiley \& Sons.

7. Bollen KA (1989). Structural Equation with Latent Variables. New York: John Wiley \& Sons.

8. Bompa TO (1999). Periodization. Theory and Methodology of Training. $4^{\text {th }}$ Edition. Champaign: Human Kinetics.

9. Bouchard C, Shephard RJ, Stephens T (eds.) (1994). Physical Activity, Fitness, and Health: International Proceedings and Consensus Statements. Champaign: Human Kinetics.

10. Cuttance P, Ecob R (1987). Structural Modeling by Example. Applications in Education, Sociological, and Behavioral Research. Cambridge: Cambridge University Press.

11. Author (1986). English Dictionary. Penguin Reference Books. Middlesex: Longman Group Limited.

12. Finkel SA (1995). Causal Analysis with Panel Data. Sage University Paper Series on Quantitative Applications in the Social Sciences, 07-105. Thousand Oakes: Sage.

13. Fouget JMB, Gallart GC (2000). Modelos de Ecuaciones Estruturales. Madrid: Editorial la Muralla, SA.

14. Foulkes MA, Davies LE (1981). An index of tracking for longitudinal data. Biometrics, 37:439-446.

15. Glenmark B, Hedberg G, Jansson E (1994). Prediction of physical activity level in adulthood by physical characteristics, physical performance and physical activity in adolescence: an 11-year follow-up study. European Journal of Applied Physiology. 69:530-538.

16. Hill PW (1987). Modeling the hierarchical structure of learning. In Cuttance P, Ecob R (eds.). Structural Modeling by Example. Cambridge: Cambridge University Press, 65-85.

17. Janz KF, Mahoney LT (1997). Three-year follow-up of changes in aerobic fitness during puberty: the Muscatine study. Research Quarterly for Exercise and Sport. 1:1-9.

18 Janz KF, Dawson JD, Mahoney LT (2000). Tracking physical fitness and physical activity from childhood to adolescence: the Muscatine study. Medicine $\mathcal{E}$ Science in Sports $\&$ Exercise. 7: 1250-1257.

19. Joreskog KG (1969). A general approach to confirmatory maximum likelihood factor analysis. Psychometrika. $34: 183-202$

20. Jöreskog KG (1970) Estimating and testing of simplex models. British Journal of Mathematical and Statistical Psychology. 23:121-145.

21. Joreskog KG, Sorbom D (1989). Lisrel 7. User's Reference Guide. Chicago: Scientific Software International.
22. Jöreskog KG, Sörbom D (1996) Lisrel 8: User's Reference Guide. Chicago: Scientific Software International.

23. Kelder S H, Berry CL, Klepp K-I, Lytle LL (1994). Longitudinal Tracking of Adolescent Smoking, Physical Activity, and Food Choice Behaviors. American Journal of Public Health. 84:1121-1126.

24. Kowalski CJ, Schneiderman ED (1992). Tracking: concepts, methods and tools. International Journal of Anthropology. 7:33-50.

25. Landis JR, Koch GG (1977). The measurement of observer agreement for categorical data. Biometrics. 37:439-446.

26. Maia JAR, Beunen G, Lefevre J, Claessens A (1998a). A estabilidade da aptidão física. O problema, essência analítica, insuficiências e apresentação de uma proposta metodológica baseada em estudos de painel com variáveis latentes. Revista Movimento. 9:58-79.

27. Maia JAR, Beunen G, Lefevre J, Claessens A (1998b). Testes empíricos a formulações desenvolvimentalistas. Um estudo centrado na modelação da estrutura de covariância. Revista Paulista de Educação Física. 2: 160-180.

28. Maia JAR, Lefevre J, Claessens A, Renson R, Vanreusel B, Beunen G (2001). Tracking of physical fitness during adolescence: a panel study in boys. Medicine \& Science in Sports E Exercise. 33:765-771.

29. Marcoulides GA, Schumacker RE (1996). Advanced Structural Equation Modeling. Issues and Techniques. Mahwah: Lawrence Erlbaum Associates.

30. McArdle JJ, Aber MS (1991) latent variable structural equations models. In Rovine MJ, von Eye A (eds.) Statistical Methods in Longitudinal Research. Volume I. Principles and Structuring Change. San Diego: Academic Press, 151-224.

31. McMahan CA (1981). An index of tracking. Biometrics. 37:447-455.

32. Muthén LK, Muthén BO (2001). Mplus Statistical Analysis with Latent Variables. User's Guide. Los Angeles: Muthén \& Muthén.

33. Paffenbarger RS, Hyde RT, Wing AL, Hsieh C-C (1986). Physical activity, all-cause mortality, and longevity of college alumni. New England Journal of Medicine. 314: 605-613.

34. Park WJ, Li J, Roche AF (1997). The decanalization of weight, recumbent length, and head circunference during infancy. American Journal of Human Biology. 9:689-698. 1997.

35. Pate RR, Baranowski T, Dowda M, Trost ST(1996). Tracking of physical activity in young children. Medicine $\mathcal{E}$ Science in Sports \& Exercise. 1:92-96.

36. Pate RR, Trost S, Dowda M, Ott AE, Ward DS, Saunders R, Felton G (1999). Tracking of physical activity, physical inactivity, and health-related physical fitness in rural youth. Pediatric Exercise Science. 11:364-376.

37. Raykov T, Marcoulides GA (2000). A First Course in Structural Equation Modeling. Mahwah: Lawrence Erlbaum Associates, Publishers.

38. Roche AF, Baumgartner RN (1987). Tracking in fat distribution during growth. In Bouchard C, Johnston, FE (eds.) Fat Distribution and Metabolic Risk Factors During Growth and Later Health Outcomes. New York: Alan R. Liss, Inc.

39. Roche AF, Guo S (1994). Tracking: its analysis and significance. Humanbiologia Budapestinensis. 25: 465-469.

40. Rosner B, Hennekens CH, Kass EH, Miall WE (1977). Age-specific correlation analysis of longitudinal blood pressure data. American Journal of Epidemiology. 4: 306-313. 
41. Schneiderman ED, Kowalski CJ (1993). Longitudinal Data Analysis. Release Notes, Version 3.2. Dallas.

42. Siff MC, Verkhoshansky Y (2000). Super Entrenamiento. Barcelona: Paidotribo.

43. Tanner JM (1978). Foetus into Man. Physical Growth from Conception to Maturity. Cambridge: Harvard University Press.

44. Taris TW (2000). A Primer in Longitudinal Data Analysis. Londres: Sage Publications.

45. Telama R, Leskinen E, Yang X (1996). Stability of habitual physical activity and sports participation: a longitudinal tracking study. Scandinavian Journal of Medicine \& Science in Sports. 6:371-378.

46. Twisk JWR, Kemper HCG, Mellenberg DJ, van Mechelen W (1996). Factors influencing tracking of cholesterol and high-density lipoprotein: the Amsterdam Growth and Health Study. Preventive Medicine. 25: 355-364.

47. U.S. Department of Health and Human Services (1996). Physical Activity and Health: A Report of the Surgeon General. Atlanta: U.S. Department of Health and Human Services, Centers for Disease Control and Prevention, National Center for Chronic Disease Control Prevention and health Promotion.

48. Van Lenthe FJ, Kemper HCG, Twisk JWR (1994). Tracking of blood pressure in children and youth. American journal of Human Biology. 6:389-399.

49. Waddington CH (1957). Strategy of the Genes: A Discussion of some Aspects of Theoretical Biology. London: Allen and Unwin.

50. Ware JH (1988) Tracking. In Kotz S, Johnson NL (eds.). Encyclopedia of Statistical Sciences. New York: John Wiley \& Sons, 302-305.

51. Ware JH, Wu MC (1981). Tracking: prediction of future values from serial measurements. Biometrics. 37:427-437.

52. Werts CE, Linn RL, Joreskog KG (1977). A simplex model for analyzing academic growth. Educational and Psychological Measurement. 37:745-756. 